\title{
Predicting Li-rich Layered Oxide Compounds as High- conductivity and Stable Solid Electrolytes
}

Wujie Qiu ${ }^{1,2}$, Tiantian Wang ${ }^{1}$, Youwei Wang ${ }^{1,2}$, Xuejun Zhou ${ }^{1,2}$, Chilin $\mathrm{Li}^{1,2}$, and

$$
\text { Jianjun } \mathrm{Liu}^{1,2,3, *}
$$

${ }^{1}$ State Key Laboratory of High Performance Ceramics and Superfine Microstructure, Shanghai Institute of Ceramics, Chinese Academy of Sciences, 1295 Dingxi Road, Shanghai 200050, China

${ }^{2}$ Center of Materials Science and Optoelectronics Engineering, University of Chinese Academy of Sciences, Beijing 100049, China

${ }^{3}$ School of Chemistry and Materials Science, Hangzhou Institute for Advanced Study, University of Chinese Academy of Science, 1 Sub-lane Xiangshan, Hangzhou 310024, China

*Email: liu@mail.sic.ac.cn 


\section{Discussion}

\section{Mechanical stability}

Elastic constants can provide information about mechanical properties and stability of compounds. The mechanical stability of crystal requires the strain energy to be positive, which implies that the whole set of elastic constants should satisfy the BornHuang criteria ${ }^{1}$.

Both $I \overline{4} 3 m$ and $P \overline{4} 3 m$ phases belong to the cubic structure, and the corresponding mechanical stability requires that the elastic stiffness constants satisfy the following conditions: $C_{11}>0, C_{44}>0,\left(C_{11-}\left|C_{12}\right|\right)>0$, and $\left(C_{11}+2 C_{12}\right)>0^{2}$. For crystal systems with lower symmetry (monoclinic $C 2 / m$ and triclinic $P \overline{1}$ structures), the mechanical stability is determined by 12 conditions $^{3,4}$,

$C_{11}>0, C_{22}>0, C_{33}>0, C_{44}>0, C_{55}>0, C_{66}>0$,

$C_{11}+C_{22}+C_{33}+2\left(C_{12}+C_{13}+C_{23}\right)>0$,

$\left(C_{33} C_{55}-C_{35}{ }^{2}\right)>0,\left(C_{44} C_{66}-C_{46}{ }^{2}\right)>0,\left(C_{22} C_{33}-2 C_{23}\right)>0$,

$C_{22}\left(C_{33} C_{55}-C_{35}{ }^{2}\right)+2 C_{23} C_{25} C_{35}-C_{23}{ }^{2} C_{55}-C_{25}{ }^{2} C_{33}>0$,

$2\left[C_{15} C_{25}\left(C_{33} C_{12}-C_{13} C_{23}\right)+\quad C_{15} C_{35}\left(C_{22} C_{13}-C_{12} C_{23}\right)+\quad C_{25} C_{35}\left(C_{11} C_{23}-C_{12} C_{13}\right)\right]-$

$\left[C_{15}{ }^{2}\left(C_{22} C_{33}-C_{23}{ }^{2}\right)+C_{25}{ }^{2}\left(C_{11} C_{33}-C_{13}{ }^{2}\right)+C_{35^{2}}\left(C_{11} C_{22}-C_{12}{ }^{2}\right)\right]+C_{55} g>0$.

where $g=C_{11} C_{22} C_{33}-C_{11} C_{23}^{2}-C_{22} C_{13}{ }^{2}-C_{33} C_{12}{ }^{2}+2 C_{12} C_{13} C_{23}$.

The elastic constants $C_{i j}$ of four proposed phases satisfy their respective mechanical stability criteria at $0 \mathrm{GPa}$, thus confirming their mechanical stability. Based on the Voigt-Reuss-Hill approximations, we calculated their bulk modulus, shear modulus, and Young's modulus, which decrease with the reduction of the coordination number of cations in the corresponding phases. This indicates that the high-coordinated configuration is beneficial to improve the structural rigidity, while the loose structure with low density has poor mechanical stability. 


\section{Figures and Tables}

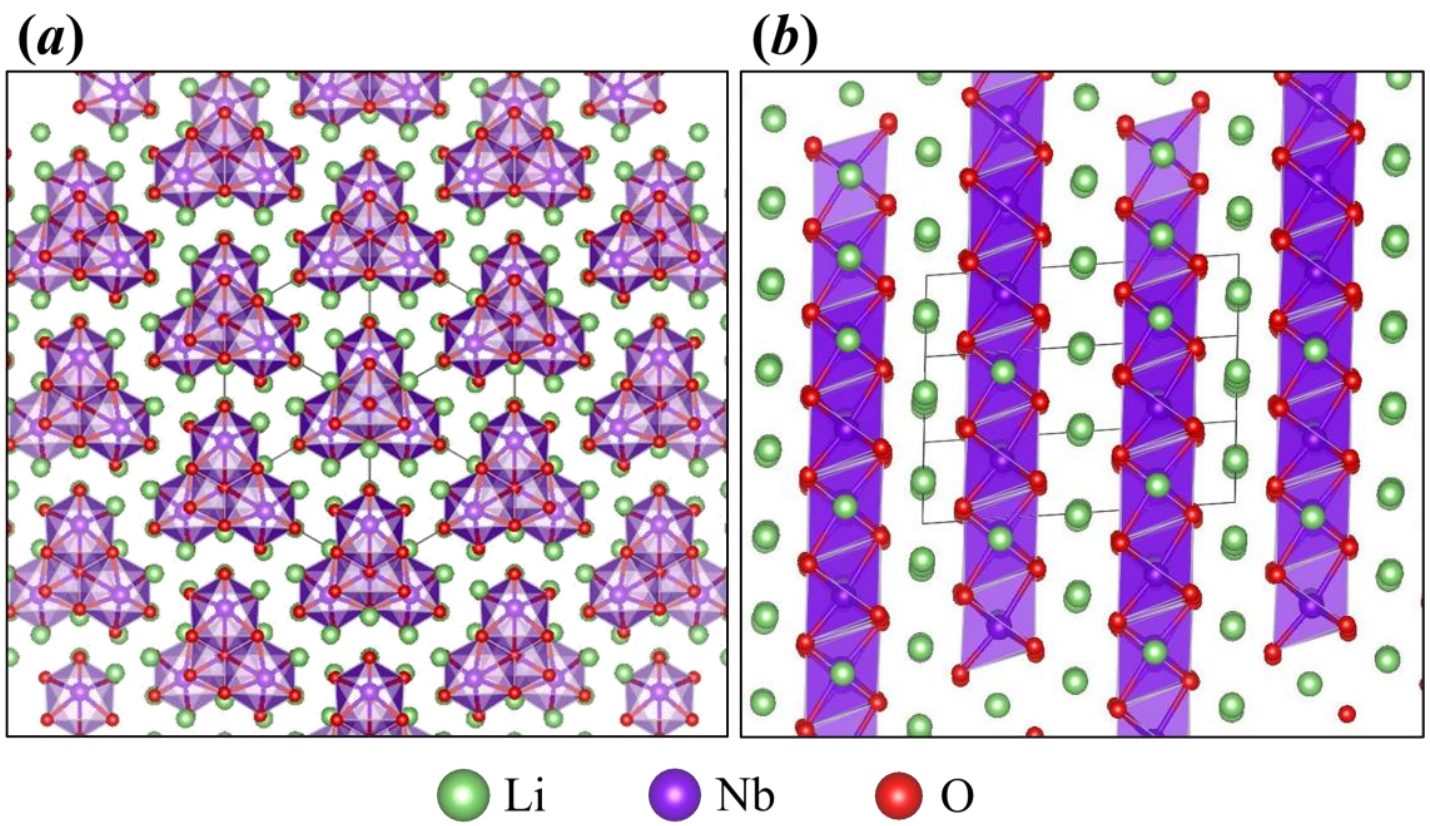

\section{Figure S1.}

Crystal structure of $\mathrm{Li}_{3} \mathrm{NbO}_{4}$ with the $I \overline{4} 3 m$ phase. (a) periodic $\mathrm{Nb}$-rich and $(b) \mathrm{Nb}$ poor regions in the structure. 

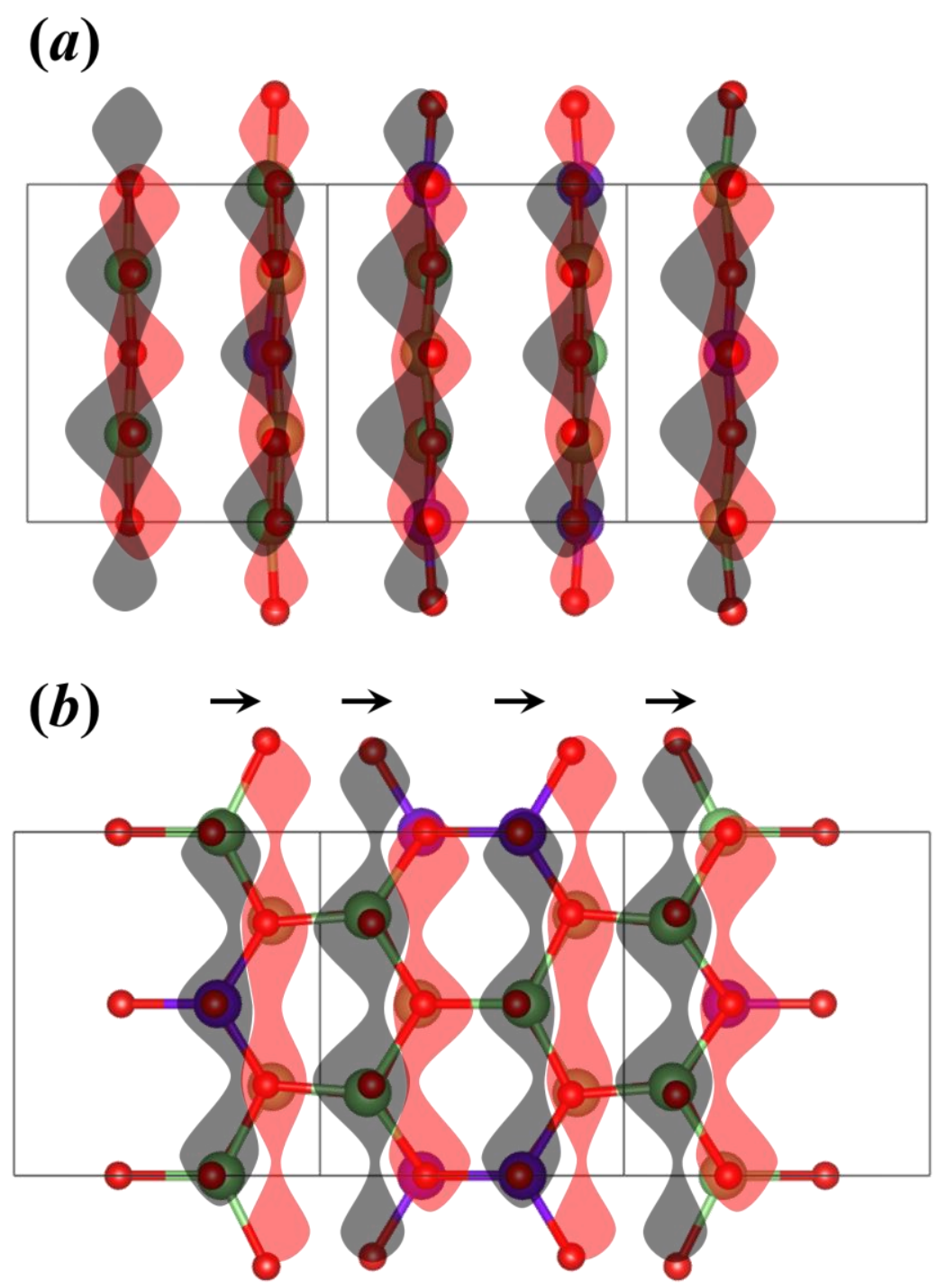

\section{Figure S2.}

Structural evolution. The atoms in (a) the six-coordinated octahedral field (marked in black) are shifted $1 / 3$ of the layer spacing to the left, transforming into $(b)$ the fivecoordinated triangular bipyramidal field. 

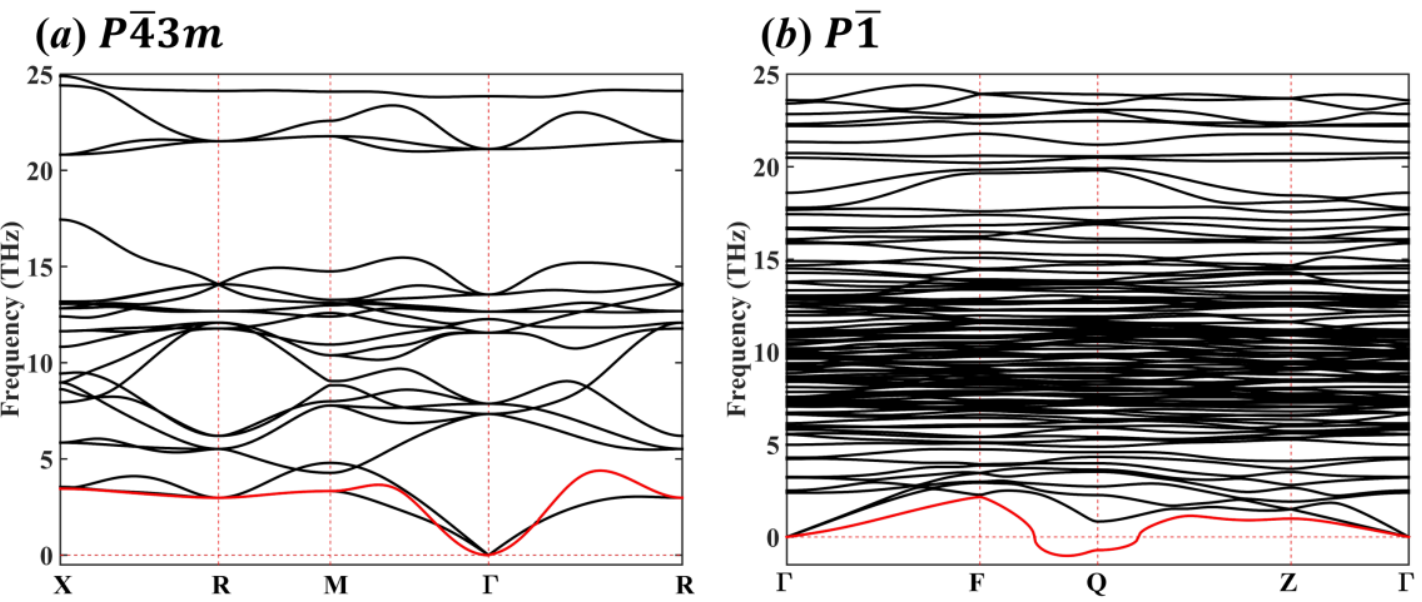

Figure S3.

Phonon dispersion curves for (a) $P \overline{4} 3 m$ and (b) $P \overline{1}$ phases of $\mathrm{Li}_{3} \mathrm{NbO}_{4}$. Soft-mode vibrations are marked in red. 
(a)

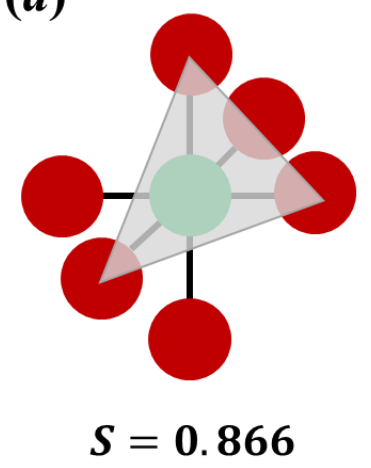

(b)

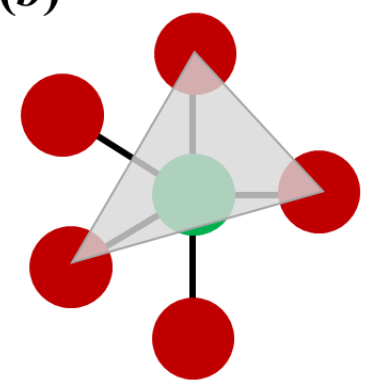

$S=1.059$ (c)

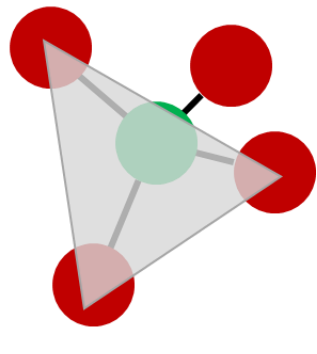

$S=1.155$

Figure S4.

By assuming the Li-O bond length as unit 1, the area of oxygen triangle in $(a)$ the hexa-, (b) penta-, and (c) tetra-coordinated structures. 
(a)

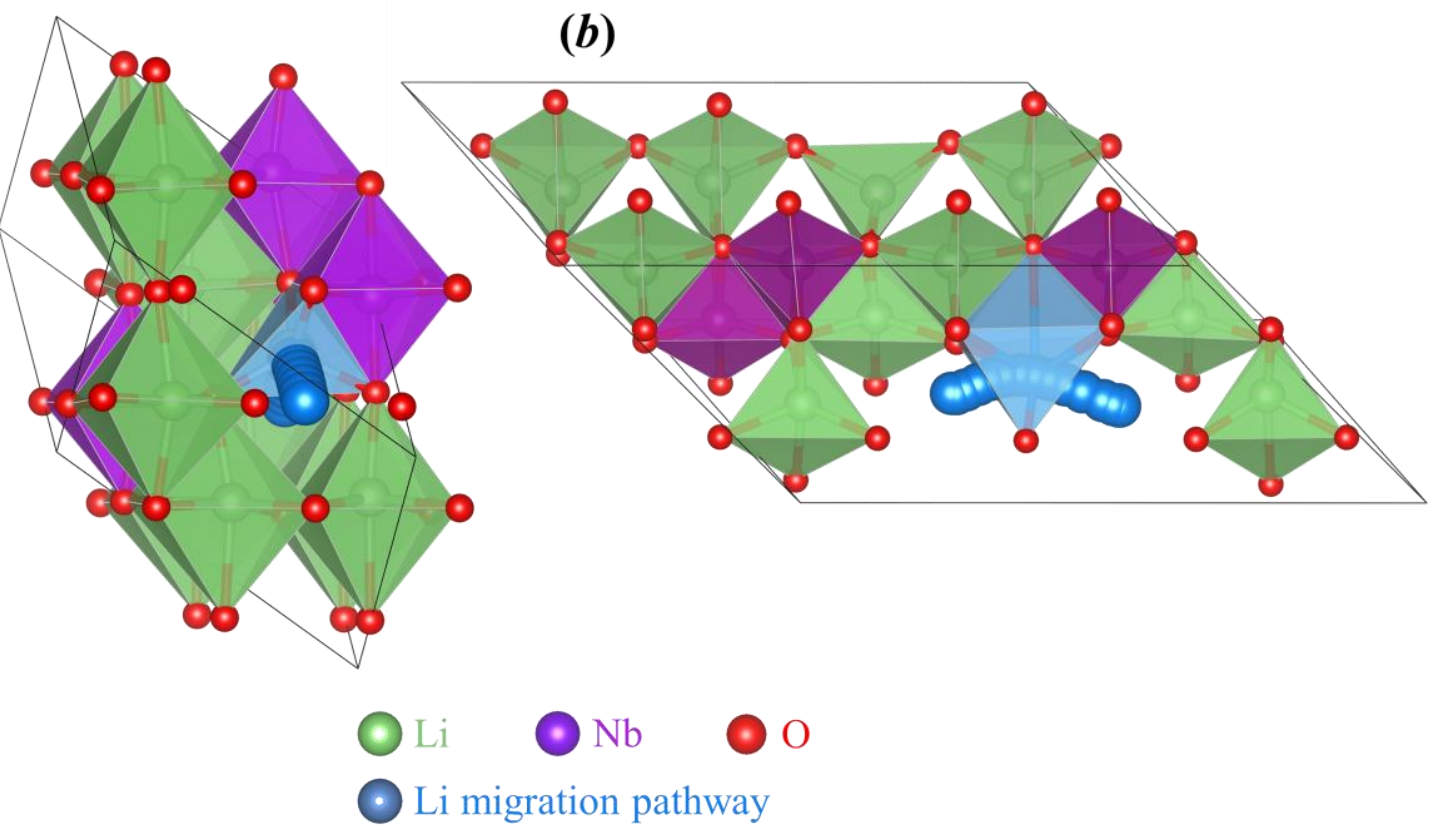

\section{Figure S5.}

Structural models of $\mathrm{Li}_{2.75} \mathrm{NbO}_{4}$ in unit cells of $(a) I \overline{4} 3 \mathrm{~m}$ and $(b) \mathrm{C} 2 / \mathrm{m}$ phases were chosen for VCNEB calculation purposes. Li migration pathways are also displayed in the two phases. 


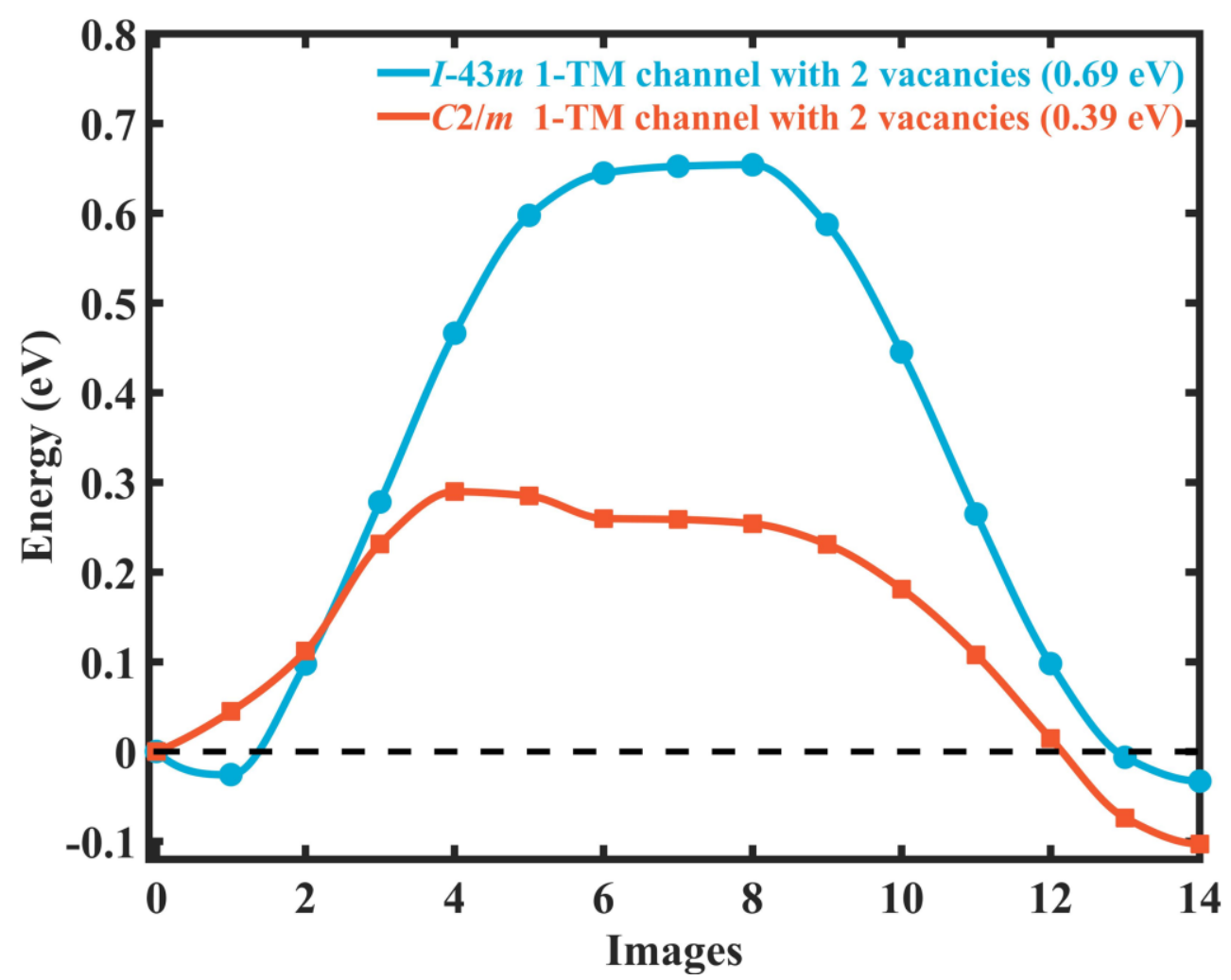

Figure S6.

Migration barriers for $\mathrm{Li}^{+}$ions in the $I \overline{4} 3 m$ and $C 2 / m$ phases. 


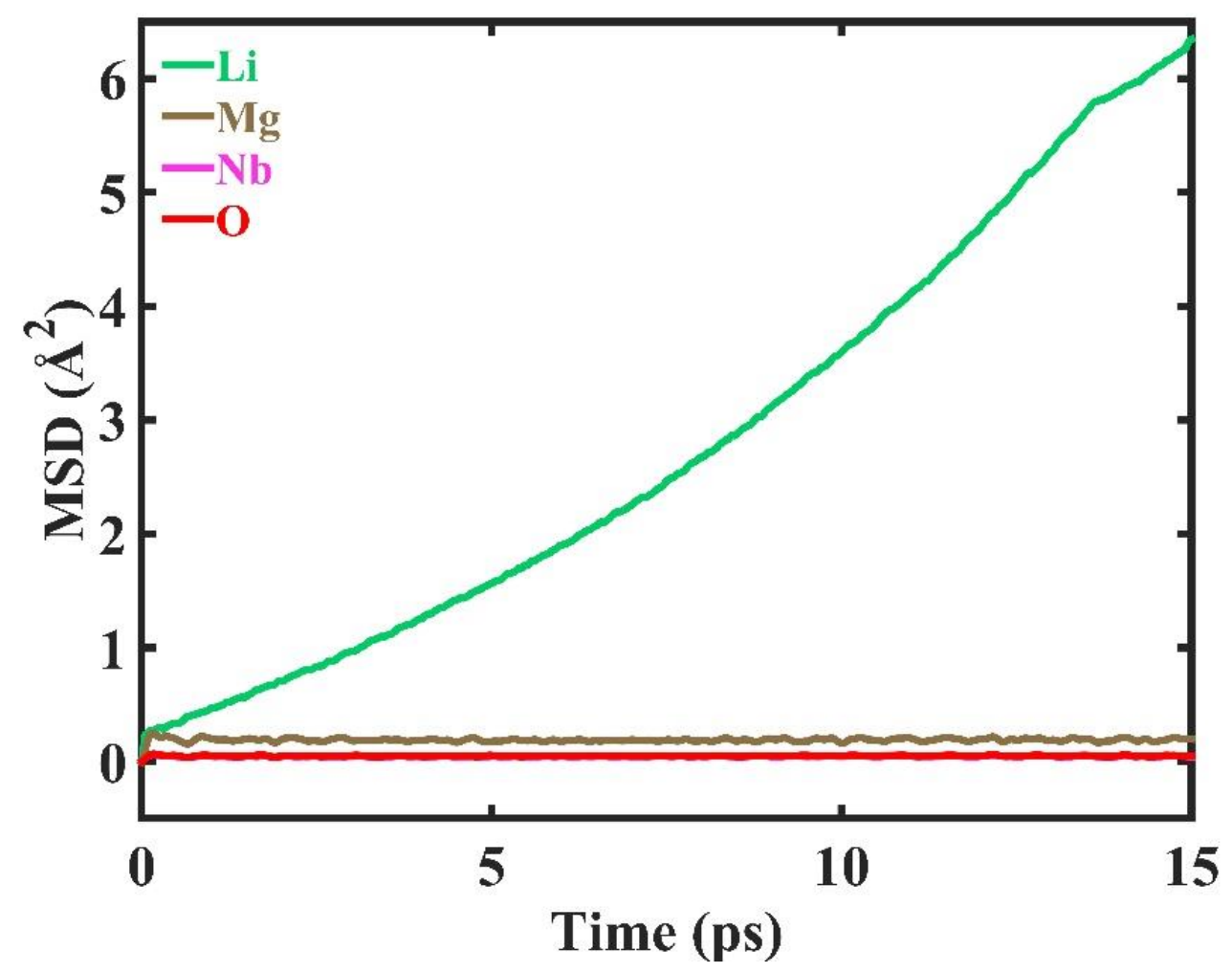

Figure S7.

Mean square displacement of $\mathrm{Li}^{+}, \mathrm{Mg}^{2+}, \mathrm{Nb}^{5+}$, and $\mathrm{O}^{2-}$ ions in $\mathrm{Li}_{2} \mathrm{Mg}_{0.5} \mathrm{NbO}_{4}$. 


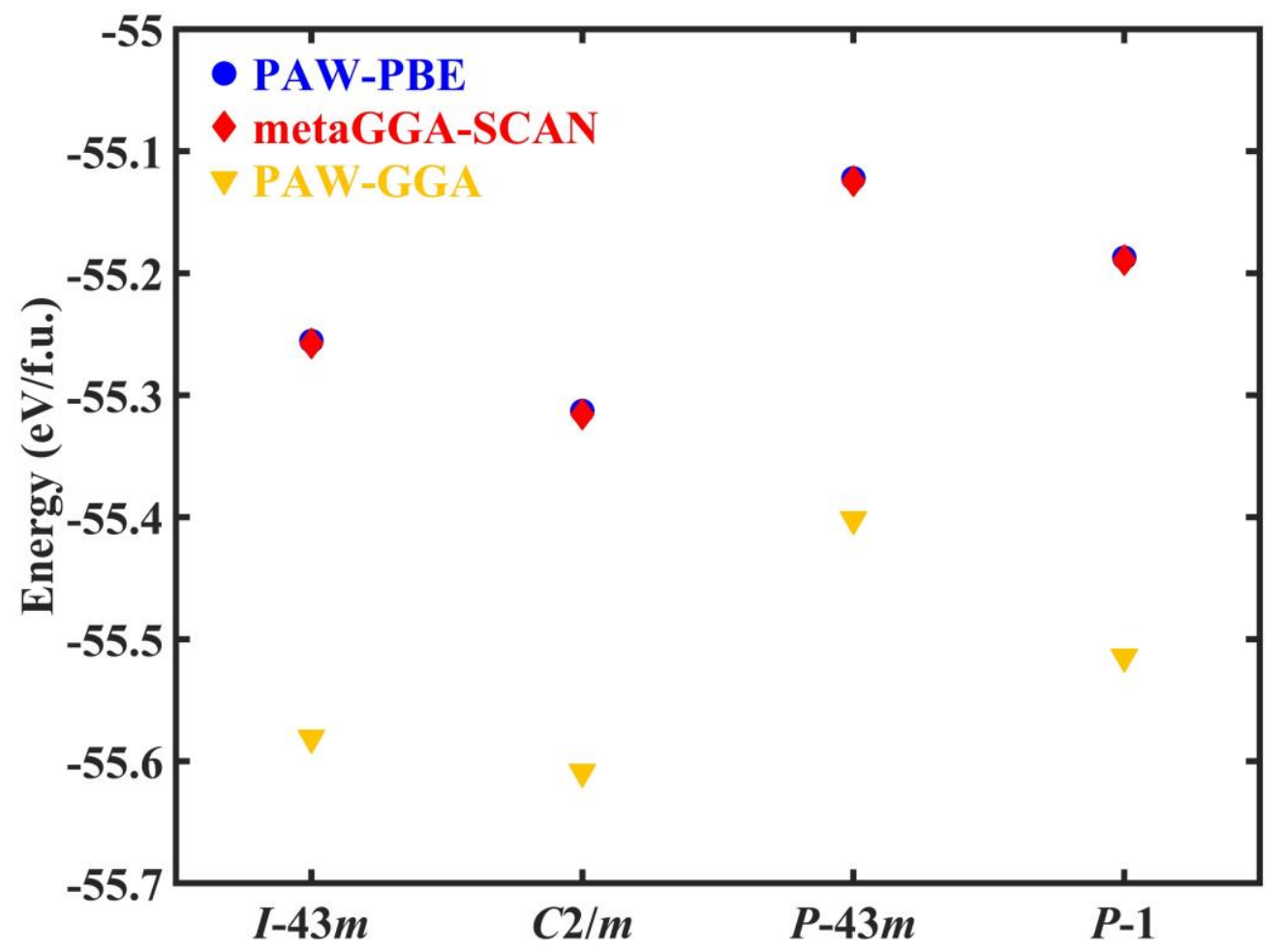

Figure S8.

Total energies of $I \overline{4} 3 m, C 2 / m, P \overline{4} 3 m$, and $P \overline{1}$ phases as predicted by PAW-PBE, metaGGA-SCAN, and PAW-GGA91 functionals. 


\section{Table S1.}

Calculated lattice parameter of the two $\mathrm{Li}_{3} \mathrm{NbO}_{4}$ phases compared with the available experimental data.

\begin{tabular}{|c|c|c|c|c|c|}
\hline Phase & $\begin{array}{l}\text { Relative energy } \\
\text { (meV/f.u.) }\end{array}$ & $a(\AA)$ & $b(\AA)$ & $c(\AA)$ & \\
\hline \multirow[t]{2}{*}{$I \overline{4} 3 m$} & 0 & 8.496 & 8.496 & 8.496 & This work \\
\hline & & 8.374 & 8.374 & 8.374 & Expt. $^{5}$ \\
\hline$C 2 / m$ & -57.44 & 6.889 & 6.889 & 7.444 & This work \\
\hline
\end{tabular}




\section{Table S2.}

Based on the Voigt-Reuss-Hill approximations, Elastic stiffness constants $C_{i j}$ (kbar), bulk modulus B (GPa), shear modulus G (GPa), Young's modulus E (GPa), and Possion's ratio $v$ of $I \overline{4} 3 m, C 2 / m, P \overline{4} 3 m$, and $P \overline{1}$ phases at ambient pressure.

\begin{tabular}{|c|c|c|c|c|c|c|c|c|c|c|c|c|c|}
\hline \multirow{6}{*}{$C_{I \overline{4} 3 m}=$} & 2073.712 & 422.966 & 422.967 & 0.001 & 0.001 & 0.001 & \multirow{6}{*}{$C_{C 2 / m}=$} & 1145.202 & 651.517 & 615.560 & 65.697 & 29.290 & 36.668 \\
\hline & 422.966 & 1922.986 & 573.694 & -213.159 & 0.002 & 0.001 & & 651.517 & 1069.467 & 567.078 & -3.192 & 5.464 & 25.021 \\
\hline & 422.967 & 573.694 & 1922.991 & 213.160 & 0.002 & 0.001 & & 615.560 & 567.078 & 1115.865 & 40.012 & 27.571 & 48.939 \\
\hline & 0.001 & -213.159 & 213.160 & 523.920 & 0.001 & 0.001 & & 65.697 & -3.192 & 40.012 & 263.895 & 4.878 & 17.942 \\
\hline & 0.001 & 0.002 & 0.002 & 0.001 & 674.648 & 213.160 & & 29.290 & 5.464 & 27.571 & 4.878 & 599.241 & 4.408 \\
\hline & 0.001 & 0.001 & 0.001 & 0.001 & 213.160 & 523.921 & & 36.668 & 25.021 & 48.939 & 17.942 & 4.408 & 604.581 \\
\hline \multirow{6}{*}{$C_{P \overline{4} 3 m}=$} & 851.317 & 467.882 & 467.882 & 0.000 & 0.000 & 0.000 & \multirow{6}{*}{$C_{P \overline{1}}=$} & 2236.246 & 633.204 & 307.120 & 19.297 & 33.431 & 218.506 \\
\hline & 467.882 & 851.317 & 467.882 & 0.000 & 0.000 & 0.000 & & 633.204 & 1864.065 & 320.699 & 25.621 & 53.326 & -215.696 \\
\hline & 467.882 & 467.882 & 851.317 & 0.000 & 0.000 & 0.000 & & 307.120 & 320.699 & 1895.415 & -0.515 & 36.606 & 20.204 \\
\hline & 0.000 & 0.000 & 0.000 & 497.538 & 0.000 & 0.000 & & 19.297 & 25.621 & -0.515 & 750.913 & -202.163 & 32.143 \\
\hline & 0.000 & 0.000 & 0.000 & 0.000 & 497.538 & 0.000 & & 33.431 & 53.326 & 36.606 & -202.163 & 428.599 & 6.276 \\
\hline & 0.000 & 0.000 & 0.000 & 0.000 & 0.000 & 497.538 & & 218.506 & -215.696 & 20.204 & 32.143 & 6.276 & 442.901 \\
\hline \multicolumn{2}{|c|}{ Space Group } & \multicolumn{4}{|c|}{ Coordination Number } & \multicolumn{2}{|r|}{$\begin{array}{c}\mathrm{B}_{\mathrm{H}} \\
(\mathrm{GPa})\end{array}$} & $\begin{array}{c}\mathbf{G}_{\mathrm{H}} \\
(\mathbf{G P a})\end{array}$ & & \multicolumn{2}{|c|}{$\begin{array}{c}\mathbf{E}_{\mathbf{H}} \\
(\mathbf{G P a})\end{array}$} & & $v$ \\
\hline \multicolumn{2}{|c|}{$I \overline{4} 3 m$} & \multicolumn{4}{|c|}{6} & \multicolumn{2}{|r|}{97.32} & 63.50 & & \multicolumn{2}{|c|}{156.47} & & 0.23 \\
\hline \multicolumn{2}{|c|}{$C 2 / m$} & \multicolumn{4}{|c|}{5} & \multicolumn{2}{|r|}{77.62} & 36.08 & & \multicolumn{2}{|c|}{93.73} & & 0.30 \\
\hline \multicolumn{2}{|c|}{$P \overline{4} 3 m$} & \multicolumn{4}{|c|}{4} & \multicolumn{2}{|r|}{59.57} & 33.95 & & \multicolumn{2}{|c|}{85.58} & & 0.26 \\
\hline \multicolumn{2}{|c|}{$P \overline{1}$} & \multicolumn{4}{|c|}{6} & \multicolumn{2}{|r|}{93.76} & 61.29 & & \multicolumn{2}{|c|}{150.97} & & 0.23 \\
\hline
\end{tabular}


Table S3.

Migration barriers of $\mathrm{Li}$ ions along the $\mathrm{ODH}$ and $\mathrm{PSH}$ paths with different concentrations.

\begin{tabular}{cccc}
\hline $\begin{array}{c}\text { Structure } \\
\text { phase }\end{array}$ & $\begin{array}{c}\text { Li-ion } \\
\text { concentration }\end{array}$ & $\begin{array}{c}\text { Migration barrier } \\
\text { in ODH path } \\
(\mathrm{eV})\end{array}$ & $\begin{array}{c}\text { Migration barrier } \\
\text { in PSH path } \\
(\mathrm{eV})\end{array}$ \\
\hline $\begin{array}{l}I \overline{4} 3 m \\
\text { phase }\end{array}$ & High $(91.6 \%)$ & 0.79 & 0.89 \\
$\begin{array}{l}C 2 / m \\
\text { phase }\end{array}$ & High $(91.6 \%)$ & 0.72 & 0.69 \\
\hline
\end{tabular}




\section{Table S4.}

The formation energy of $\mathrm{Li}_{3} \mathrm{NbO}_{4}$ structures doped with different $\mathrm{Mg}$ concentrations.

The formula for the formation energy can be written as

$$
\Delta E_{\mathrm{Li}_{12-2 x} \mathrm{TM}_{x} \mathrm{Nb}_{4} \mathrm{O}_{16}}=E_{\mathrm{Li}_{12-2 x} \mathrm{TM}_{x} \mathrm{Nb}_{4} \mathrm{O}_{16}}-\left((6-x) E_{\mathrm{Li}_{2} \mathrm{O}}+x E_{\mathrm{TMO}}+2 E_{\mathrm{Nb}_{2} \mathrm{O}_{5}}\right)
$$

\begin{tabular}{ccc}
\hline Number of each atom in a cell & $\begin{array}{c}\text { Simplified chemical } \\
\text { formula }\end{array}$ & $\begin{array}{c}\text { Formation energy } \\
\text { (eV/atom) }\end{array}$ \\
\hline $\mathrm{Li}_{10} \mathrm{MgNb}_{4} \mathrm{O}_{16}$ & $\mathrm{Li}_{2.5} \mathrm{Mg}_{0.25} \mathrm{NbO}_{4}$ & -0.22 \\
$\mathrm{Li}_{8} \mathrm{Mg}_{2} \mathrm{Nb}_{4} \mathrm{O}_{16}$ & $\mathrm{Li}_{2} \mathrm{Mg}_{0.5} \mathrm{NbO}_{4}$ & -0.23 \\
$\mathrm{Li}_{6} \mathrm{Mg}_{3} \mathrm{Nb}_{4} \mathrm{O}_{16}$ & $\mathrm{Li}_{1.5} \mathrm{Mg}_{0.75} \mathrm{NbO}_{4}$ & -0.19 \\
$\mathrm{Li}_{4} \mathrm{Mg}_{4} \mathrm{Nb}_{4} \mathrm{O}_{16}$ & $\mathrm{LiMgNbO}_{4}$ & -0.13 \\
$\mathrm{Li}_{2} \mathrm{Mg}_{5} \mathrm{Nb}_{4} \mathrm{O}_{16}$ & $\mathrm{Li}_{0.5} \mathrm{Mg}_{1.25} \mathrm{NbO}_{4}$ & -0.06 \\
\hline
\end{tabular}




\section{Table S5.}

Formation energy of Li-ion vacancies.

The formation energy of Li-ion vacancies ( $\left.\Delta E_{\text {undoped }}\right)$ in $\mathrm{Li}_{3} \mathrm{NbO}_{4}$ can be expressed as

$$
\Delta E_{\text {undoped }}=E\left(\mathrm{Li}_{2.5} \square_{0.5} \mathrm{NbO}_{4}\right)+0.5 E(\mathrm{Li})-E\left(\mathrm{Li}_{3} \mathrm{NbO}_{4}\right)
$$

where $E\left(\mathrm{Li}_{2.5} \square_{0.5} \mathrm{NbO}_{4}\right), \quad E\left(\mathrm{Li}_{3} \mathrm{NbO}_{4}\right)$, and $E(\mathrm{Li})$ are the total energies of $\mathrm{Li}_{2.5} \square_{0.5} \mathrm{NbO}_{4}, \mathrm{Li}_{3} \mathrm{NbO}_{4}$, and Li metal, respectively. The formation energy of Li-ion vacancies $\left(\Delta E_{\mathrm{Mg}^{2+}}\right.$ doped $)$ in $\mathrm{Li}_{2} \mathrm{Mg}_{0.5} \mathrm{NbO}_{4}$ can be expressed as

$$
\Delta E_{\mathrm{Mg}^{2+} \text { doped }}=E\left(\mathrm{Li}_{2} \square 0.5 \mathrm{Mg}_{0.5} \mathrm{NbO}_{4}\right)+0.5 E(\mathrm{Li})-E\left(\mathrm{Li}_{2.5} \mathrm{Mg}_{0.5} \mathrm{NbO}_{4}\right)
$$

where $E\left(\mathrm{Li}_{2} \square 0.5 \mathrm{Mg}_{0.5} \mathrm{NbO}_{4}\right)$ and $E\left(\mathrm{Li}_{2.5} \mathrm{Mg}_{0.5} \mathrm{NbO}_{4}\right)$ are the total energies of $\mathrm{Li}_{2} \square$ ${ }_{0.5} \mathrm{Mg}_{0.5} \mathrm{NbO}_{4}$ and $\mathrm{Li}_{2.5} \mathrm{Mg}_{0.5} \mathrm{NbO}_{4}$, respectively.

\begin{tabular}{cc}
\hline Compound & $\begin{array}{c}\text { Formation energy } \\
\text { (eV/f.u.) }\end{array}$ \\
\hline $\mathrm{Li}_{2.5} \square_{0.5} \mathrm{NbO}_{4}$ & 2.48 \\
$\mathrm{Li}_{2} \square_{0.5} \mathrm{Mg}_{0.5} \mathrm{NbO}_{4}$ & 0.56 \\
\hline
\end{tabular}


Table S6.

Structural parameters for the $I \overline{4} 3 m, C 2 / m, P \overline{4} 3 m$, and $P \overline{1}$ phases.

\begin{tabular}{|c|c|c|c|c|c|}
\hline Phase & $\begin{array}{c}\text { Lattice } \\
\text { parameter }\end{array}$ & Atom & $x / a$ & $y / b$ & $z / c$ \\
\hline \multirow{4}{*}{$I \overline{4} 3 m$} & \multirow{4}{*}{$a=b=c=8.496 \AA$} & $\mathrm{Li}$ & 0.270 & 0.514 & 0.000 \\
\hline & & $\mathrm{Nb}$ & 0.720 & 0.000 & 0.000 \\
\hline & & $\mathrm{O} 1$ & 0.226 & 0.000 & 0.000 \\
\hline & & $\mathrm{O} 2$ & 0.238 & 0.238 & 0.754 \\
\hline \multirow{6}{*}{$C 2 / m$} & \multirow{6}{*}{$\begin{array}{c}a=8.788 \AA \\
b=6.762 \AA \\
c=7.444 \AA \\
\alpha=\gamma=90^{\circ} \\
\beta=126.266^{\circ}\end{array}$} & Li1 & 0.049 & 0.259 & 0.842 \\
\hline & & $\mathrm{Li} 2$ & 0.184 & 0.000 & 0.677 \\
\hline & & $\mathrm{Nb}$ & 0.230 & 0.500 & 0.667 \\
\hline & & $\mathrm{O} 1$ & 0.286 & 0.264 & 0.826 \\
\hline & & $\mathrm{O} 2$ & 0.464 & 0.000 & 0.650 \\
\hline & & $\mathrm{O} 3$ & 0.441 & 0.500 & 0.658 \\
\hline \multirow{3}{*}{$P \overline{4} 3 m$} & \multirow{3}{*}{$a=b=c=4.556 \AA$} & $\mathrm{Li}$ & 0.000 & 0.000 & 0.500 \\
\hline & & $\mathrm{Nb}$ & 0.500 & 0.500 & 0.500 \\
\hline & & $\mathrm{O}$ & 0.263 & 0.263 & 0.737 \\
\hline \multirow{17}{*}{$P \overline{1}$} & \multirow{17}{*}{$\begin{array}{l}a=5.173 \AA \\
b=6.049 \AA \\
c=9.959 \AA \\
\alpha=81.521^{\circ} \\
\beta=95.165^{\circ} \\
\gamma=90.198^{\circ}\end{array}$} & Li1 & 0.376 & 0.183 & 0.249 \\
\hline & & Li2 & 0.127 & 0.560 & 0.751 \\
\hline & & Li3 & 0.729 & 0.135 & 0.497 \\
\hline & & $\mathrm{Li} 4$ & 0.772 & 0.635 & 0.503 \\
\hline & & Li5 & 0.983 & 0.735 & 0.000 \\
\hline & & Li6 & 0.500 & 0.500 & 0.000 \\
\hline & & Li7 & 0.500 & 0.000 & 0.000 \\
\hline & & $\mathrm{Nb} 1$ & 0.877 & 0.973 & 0.250 \\
\hline & & $\mathrm{Nb} 2$ & 0.376 & 0.652 & 0.251 \\
\hline & & $\mathrm{O} 1$ & 0.825 & 0.049 & 0.861 \\
\hline & & $\mathrm{O} 2$ & 0.077 & 0.671 & 0.364 \\
\hline & & $\mathrm{O} 3$ & 0.578 & 0.894 & 0.364 \\
\hline & & $\mathrm{O} 4$ & 0.327 & 0.272 & 0.863 \\
\hline & & O5 & 0.047 & 0.148 & 0.370 \\
\hline & & O6 & 0.547 & 0.416 & 0.369 \\
\hline & & O7 & 0.798 & 0.521 & 0.872 \\
\hline & & O8 & 0.298 & 0.796 & 0.870 \\
\hline
\end{tabular}




\section{Reference}

(1) Le Page, Y.; Saxe, P., Symmetry-general least-squares extraction of elastic data for strained materials fromab initiocalculations of stress. Phys. Rev. B 2002, 65, 104104.

(2) Wu, Z.; Hao, X.; Liu, X.; Meng, J., Structures and elastic properties of $\mathrm{OsN}_{2}$ investigated via first-principles density functional calculations. Phys. Rev. B 2007, $75,054115$.

(3) Wu, Z.-j.; Zhao, E.-j.; Xiang, H.-p.; Hao, X.-f.; Liu, X.-j.; Meng, J., Crystal structures and elastic properties of superhard $\mathrm{IrN}_{2}$ and $\mathrm{IrN}_{3}$ from first principles. Phys. Rev. B 2007, 76, 054115.

(4) Liao, Z.; Huai, P.; Qiu, W.; Ke, X.; Zhang, W.; Zhu, Z., Lattice dynamics and lattice thermal conductivity of thorium dicarbide. J. Nucl. Mater. 2014, 454, 142148.

(5) Yabuuchi, N.; Takeuchi, M.; Nakayama, M.; Shiiba, H.; Ogawa, M.; Nakayama, K.; Ohta, T.; Endo, D.; Ozaki, T.; Inamasu, T.; Sato, K.; Komaba, S., Highcapacity electrode materials for rechargeable lithium batteries: $\mathrm{Li}_{3} \mathrm{NbO}_{4}$-based system with cation-disordered rocksalt structure. Proc. Natl. Acad. Sci. USA 2015, $112,7650-5$. 